\title{
Child abuse and mental disorders in Canada
}

\author{
Tracie O. Afifi PhD, Harriet L. MacMillan MD, Michael Boyle PhD, Tamara Taillieu MSc, Kristene Cheung BA, \\ Jitender Sareen MD
}

See also humanities article, www.cmaj.ca/lookup/doi/10.1503/cmaj.140314

Competing interests: None declared.

This article has been peer reviewed.

Correspondence to:

Tracie Afifi, tracie.afifi@

med.umanitoba.ca

CMAJ 2014. DOI:10.1503

/cmaj.131792

\begin{abstract}
Background: Nationally representative Canadian data on the prevalence of child abuse and its relation with mental disorders are lacking. We used contemporary, nationally representative data to examine the prevalence of 3 types of child abuse (physical abuse, sexual abuse and exposure to intimate partner violence) and their association with 14 mental conditions, including suicidal ideation and suicide attempts.
\end{abstract}

Methods: We obtained data from the 2012 Canadian Community Health Survey: Mental Health, collected from the 10 provinces. Respondents aged 18 years and older were asked about child abuse and were selected for the study sample ( $n=23$ 395). The survey had a multistage stratified cluster design (household response rate $79.8 \%$ ).

Results: The prevalence of any child abuse was $32 \%$ (individual types ranged from $8 \%$ to $26 \%$ ). All types of child abuse were associated

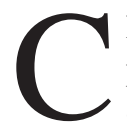

hild abuse is a major public health problem worldwide with serious adverse effects at later ages. The best estimates of the prevalence of child abuse in Canada come from 2 representative samples from Ontario, 1 from 1990 (Ontario Health Supplement, $n=9953$, age $\geq 15 \mathrm{yr})^{1}$ and 1 from 2000-2001 (Ontario Child Health Study, $n=1893$, age $21-35 \mathrm{yr}$ ). ${ }^{2}$ The 1990 study indicated that $31 \%$ of males and $21 \%$ of females had experienced physical child abuse, and $4 \%$ of males and $13 \%$ of females had experienced sexual child abuse. ${ }^{1}$ Similar trends were noted in data collected in 2000-2001. ${ }^{2}$ Prevalence of sexual abuse was found to be higher in the Ontario Child Health Study, but this was thought to be related to the inclusion of sexual abuse committed by anyone (e.g., adolescents), rather than only adults, as was the case with the Ontario Health Supplement. ${ }^{1,2}$ Data from Quebec collected in 2006 indicated that $23 \%$ of men and $17 \%$ of women had experienced physical child abuse; $10 \%$ of men and $22 \%$ of with all mental conditions, including suicidal ideation and suicide attempts, after adjustment for sociodemographic variables (adjusted odds ratios ranged from 1.4 to 7.9). We found a dose-response relation, with increasing number of abuse types experienced corresponding with greater odds of mental conditions. Associations between child abuse and attention deficit disorder, suicidal ideation and suicide attempts showed stronger effects for women than men.

Interpretation: We found robust associations between child abuse and mental conditions. Health care providers, especially those assessing patients with mental health problems, need to be aware of the relation between specific types of child abuse and certain mental conditions. Success in preventing child abuse could lead to reductions in the prevalence of mental disorders, suicidal ideation and suicide attempts.

women had experienced sexual child abuse; and $22 \%$ of men and women had experienced psychological child abuse, including exposure to intimate partner violence. ${ }^{3,4}$ Unfortunately, these estimates are dated, restricted to only 2 provinces, based on limited assessments of child abuse and, in the case of the Quebec data, involve a low response rate of $30 \%$.

Although the association between child abuse and subsequent mental conditions (including disorders, suicidal ideation and suicide attempts) is well established, ${ }^{5-9}$ we know little about differential effects associated with several abuse types and specific mental disorders, and we currently have no nationally representative Canadian data. Filling this gap in knowledge would produce evidence-based Canadian findings that could inform policy, prevention and intervention efforts specifically aimed at reducing both child abuse and poor mental health outcomes.

Our overall objective was to extend our understanding of the relation between child abuse and mental conditions (i.e., mental disorders, suicidal 
ideation and suicide attempts) $)^{6-8,10-19}$ with a more detailed examination of child abuse severity and mental health outcomes. We used a nationally representative Canadian sample to examine 3 types of child abuse (physical abuse, sexual abuse and exposure to intimate partner violence) and 14 mental conditions, including suicidal ideation and suicide attempts. The specific research objectives were to estimate the prevalence of 3 types of physical abuse, sexual abuse and exposure to intimate partner violence; to examine the association of mental conditions with types of child abuse and number of child abuse types experienced; and to determine whether sex differences exist in the associations between child abuse and mental conditions.

\section{Methods}

\section{Data and sample}

We obtained data from the master file of the 2012 Canadian Community Health Survey: Mental Health. ${ }^{20}$ The 2012 Canadian Community Health Survey was a cross-sectional study that used a multistage stratified cluster sampling design: it included a representative sample of respondents aged 15 years and older living in the 10 provinces $(n=25113)$ and excluded residents in the 3 territories, residents in indigenous communities, full-time members of the Canadian Forces and people living in institutions (collectively less than $3 \%$ of the Canadian population). ${ }^{20}$ Most interviews (87\%) were conducted in person by trained lay interviewers using computer-assisted interviewing. ${ }^{20}$ The overall household-level response rate was $79.8 \%$, and the household and person response rate was $68.9 \% .{ }^{20}$ Because of the sensitive nature of the items, the questions about child abuse were only asked for respondents aged 18 years and older $(n=23395)$. Respondents were informed about privacy, confidentially and the voluntary nature of the survey, and gave their consent to participate. ${ }^{21}$

\section{Primary measurements}

\section{Child abuse}

Physical abuse, sexual abuse and exposure to intimate partner violence were assessed in the 2012 Canadian Community Health Survey using items from the Childhood Experiences of Violence Questionnaire, a valid and reliable tool developed for assessing youth victimization. ${ }^{22}$ All respondents aged 18 years and older were asked to answer questions about childhood abuse that occurred before the age of 16 years. Responses to questions about child abuse were scored on an ordinal scale representing frequency of occurrence (never, 1-2 times, 3-5 times, 6-10 times or $>10$ times). In this study, binary classifications of child abuse (present/absent) followed the guidelines of the Childhood Experiences of Violence Questionnaire as follows. Physical abuse was classified as 1 or more of the following 3 criteria: 1) being slapped on the face, head or

Table 1: Prevalence of child abuse in Canada, by type

\begin{tabular}{|c|c|c|c|c|}
\hline \multirow[b]{2}{*}{ Variable } & \multicolumn{3}{|c|}{ No. $(\%) *$} & \multirow[b]{2}{*}{$p$ valuet } \\
\hline & Whole sample & Men & Women & \\
\hline \multicolumn{5}{|l|}{ Physical abuse } \\
\hline Slapped on face head or ears, hit or spanked & $5080 \quad(22.3)$ & $2700 \quad(26.0)$ & $(18.6)$ & $<0.001$ \\
\hline Pushed, grabbed, shoved, something thrown at & $2550 \quad(10.5)$ & $1300 \quad(11.8)$ & 1250 & $<0.001$ \\
\hline Kicked, bit, punched, choked, burned, attacked & $2450 \quad(9.9)$ & $1350 \quad(11.9)$ & 1100 & $<0.001$ \\
\hline Any physical abuse & $6070 \quad(26.1)$ & $3280 \quad(31.0)$ & $(21.3)$ & $<0.001$ \\
\hline Sexual abuse & $2760 \quad(10.1)$ & $(5.8)$ & $(14.4)$ & $<0.001$ \\
\hline Exposure to intimate partner violence & $1900 \quad(7.9)$ & $(6.9)$ & 1170 & $<0.001$ \\
\hline Any child abuse & $7700 \quad(32.1)$ & $3610 \quad(34.0)$ & $4090 \quad(30.3)$ & $<0.001$ \\
\hline \multicolumn{5}{|l|}{ Number of child abuse types } \\
\hline No abuse & $15690 \quad(68.0)$ & $6890 \quad(66.1)$ & $(69.8)$ & $<0.001$ \\
\hline 1 type only & $5260 \quad(22.3)$ & $2720 \quad(25.8)$ & $(19.0)$ & $<0.001$ \\
\hline 2 types & 1790 & $720 \quad(6.6)$ & 1070 & $<0.001$ \\
\hline 3 types & $(2.4)$ & $(1.5)$ & $(3.2)$ & $<0.001$ \\
\hline
\end{tabular}


ears, or hit or spanked with something hard 3 or more times; 2) being pushed, grabbed or shoved, or having something thrown at the respondent to hurt them 3 or more times; and 3) being kicked, bit, punched, choked, burned or physically attacked 1 or more times. Sexual abuse was clas- sified as experiencing attempts or being forced into unwanted sexual activity by being threatened, held down or hurt in some way, and/or sexually touched meaning unwanted touching or grabbing, kissing or fondling against the respondent's will 1 or more times. Exposure to intimate

Table 2: Sociodemographic variables by child abuse in Canada

\begin{tabular}{|c|c|c|c|}
\hline \multirow[b]{2}{*}{ Variable } & \multicolumn{2}{|c|}{ No. $(\%) *$} & \multirow[b]{2}{*}{ OR $(95 \% \mathrm{Cl})$} \\
\hline & No child abuse & Child abuse & \\
\hline \multicolumn{4}{|l|}{ Age, yr } \\
\hline $18-34$ & 4480 (74.6) & $1690 \quad(25.4)$ & 1.0 \\
\hline $35-49$ & 3060 (62.9) & $1955 \quad(37.1)$ & $1.7(1.5-2.0)$ \\
\hline $50-64$ & $3830 \quad(62.5)$ & $2475 \quad(37.5)$ & $1.8(1.5-2.0)$ \\
\hline$\geq 65$ & 4330 (72.9) & $1580 \quad(27.1)$ & $1.1(0.96-1.2)$ \\
\hline \multicolumn{4}{|l|}{ Education } \\
\hline No high school diploma & 2880 (70.9) & $1225 \quad(29.1)$ & 1.0 \\
\hline High school diploma & $2730(70.4)$ & $1180 \quad(29.6)$ & $1.0(0.9-1.2)$ \\
\hline Some postsecondary education & $960 \quad(62.3)$ & $540 \quad(37.7)$ & $1.5(1.2-1.8)$ \\
\hline Trade/college/university certificate/diploma & $5865 \quad(67.2)$ & $3090 \quad(32.8)$ & $1.2(1.0-1.4)$ \\
\hline University degree (Bachelor's or higher) & $3205(66.8)$ & $1635 \quad(33.2)$ & $1.2(1.0-1.4)$ \\
\hline \multicolumn{4}{|l|}{ Income, \$ } \\
\hline$\leq 29999$ & $3760 \quad(67.2)$ & 1995 (32.8) & 1.0 \\
\hline $30000-49999$ & 3445 (68.9) & $1515 \quad(31.1)$ & $0.9(0.8-1.1)$ \\
\hline $50000-79999$ & $3710(70.2)$ & $1765 \quad(29.8)$ & $0.9(0.8-0.99)$ \\
\hline$\geq 80000$ & $4780 \quad(66.2)$ & $2425 \quad$ (33.8) & $1.0(0.9-1.2)$ \\
\hline \multicolumn{4}{|l|}{ Marital status } \\
\hline Married/common-law & 8310 (66.6) & $4060 \quad(33.4)$ & 1.0 \\
\hline Never married & $4120 \quad(72.7)$ & $1860 \quad(27.3)$ & $0.8(0.7-0.8)$ \\
\hline Separated/divorced/widowed & 3245 (65.5) & 1760 & $1.1(0.9-1.2)$ \\
\hline \multicolumn{4}{|l|}{ Visible minority } \\
\hline No & 13225 (67.3) & $6360 \quad(32.7)$ & 1.0 \\
\hline Yes & 2435 (69.7) & $1315 \quad(30.3)$ & $0.9(0.8-1.0)$ \\
\hline \multicolumn{4}{|l|}{ Country of birth } \\
\hline Canada & 12770 (66.4) & $6360 \quad(33.6)$ & 1.0 \\
\hline Other than Canada & 2865 (71.7) & $1300 \quad(28.3)$ & $0.8(0.7-0.9)$ \\
\hline \multicolumn{4}{|l|}{ Province of residence } \\
\hline Manitoba & $1060 \quad(60.0)$ & $635 \quad(40.0)$ & 1.0 \\
\hline British Columbia & 1725 (64.2) & $1125 \quad$ (35.8) & $0.8(0.7-1.0)$ \\
\hline Alberta & 1605 (63.9) & $990 \quad(36.1)$ & $0.8(0.7-1.0)$ \\
\hline Saskatchewan & $1070 \quad(68.7)$ & $480 \quad(31.3)$ & $0.7(0.5-0.9)$ \\
\hline Ontario & $3440 \quad(68.0)$ & $1670 \quad(32.0)$ & $0.7(0.6-0.9)$ \\
\hline Quebec & $2890 \quad(72.2)$ & $1190 \quad(27.8)$ & $0.6(0.5-0.7)$ \\
\hline New Brunswick & $1030 \quad(66.2)$ & $515 \quad(33.8)$ & $0.8(0.6-0.9)$ \\
\hline Nova Scotia & $1080 \quad(66.9)$ & $535 \quad(33.1)$ & $0.7(0.6-0.9)$ \\
\hline Prince Edward Island & 755 (70.7) & $270 \quad(29.3)$ & $0.6(0.5-0.8)$ \\
\hline Newfoundland and Labrador & $1040 \quad(79.5)$ & $290 \quad(20.5)$ & $0.4(0.3-0.5)$ \\
\hline
\end{tabular}


partner violence was classified as having seen or heard parents, step-parents or guardians hitting each other or another adult in the home 3 or more times. ${ }^{22}$ We also computed variables to classify any child abuse (1 or more individual types of child abuse) and a count of the number of child abuse types (ranging from 0 to 3 ).

\section{Sociodemographic covariates}

The sociodemographic variables included as covariates in the logistic regression models were as follows: age, sex, visible minority, Canadian born, highest level of education, past year household income, marital status and province of residence.

\section{Mental health variables}

\section{Mental disorders}

Lifetime diagnoses of several axis I disorders were made using the World Health Organization version of the Composite International Diagnostic Interview and based on the criteria of the Diagnostic and Statistical Manual of Mental Disorders, 4th edition. ${ }^{23,24}$ The specific mental disorders included depression, bipolar disorder, generalized anxiety disorder, alcohol abuse/dependence, and drug abuse/dependence (including nonmedical sedatives or tranquilizers, nonmedical analgesics, nonmedical stimulants, cannabis, cocaine, club drugs such as ecstasy or ketamine, hallucinogens, heroin or opium, inhalant or solvents, and other drugs). Respondents meeting criteria for 1 or more of these disorders were classified as having any mental disorder. Additional mental disorders were assessed using self-reports by asking the respondent if they had a long-term health condition diagnosed by a health professional that had lasted or was expected to last 6 months or longer (including obsessive-compulsive disorder, posttraumatic stress disorder, panic disorder, phobia, a learning disability, attention deficit disorder and an eating disorder). Respondents who reported any of these mental conditions were classified as having 1 or more of these conditions.

\section{Suicidal ideation and suicide attempts}

Suicidal ideation was assessed with a question that asked if the respondent ever seriously thought about committing suicide or taking his or her own life (yes or no). Suicide attempts were assessed with a question asking if the respondent had ever attempted suicide or tried to take his or her own life (yes or no).

\section{Statistical analysis}

We applied statistical weights to ensure that the estimates reflected the general Canadian popula- tion. We used bootstrapping as a variance estimation technique to account for the complex survey design. First, we estimated the overall and then sex-specific prevalence of child abuse. Second, we determined the distribution of sociodemographic variables by any child abuse. Third, we computed logistic regression models to quantify the associations between child abuse and mental conditions. Models were first adjusted for sociodemographic variables (adjusted odds ratio [OR] 1) and then further adjusted for other types of child abuse and other interview-diagnosed mental disorders (adjusted OR 2). Fourth, logistic regression models were computed to examine the dose-response relation between the number of child abuse types and mental conditions adjusting for sociodemographic variables. Finally, we tested for interactions between sex and the different child abuse types, adjusting for sociodemographic variables, for each mental disorder and for suicidal ideation and suicide attempts. Significant models were determined with $95 \%$ confidence intervals (CIs) or $p<0.05$.

\section{Results}

The prevalence of any of the 3 types of child abuse was $32.1 \%$, with physical abuse being most common $(26.1 \%)$, followed by sexual abuse $(10.1 \%)$ and exposure to intimate partner violence $(7.9 \%)$ (Table 1). Women were more likely than men to have experienced sexual abuse (14.4\% v. $5.8 \%, p<0.001)$ and exposure to intimate partner violence $(8.9 \%$ v. $6.9 \%, p<0.001)$ as children. Men were more likely than women to have experienced physical child abuse $(31.0 \%$ v. $21.3 \%, p<0.001)$ and any child abuse $(34.0 \%$ v. $30.3 \%, p<0.001)$. Table 2 reports the distribution of sociodemographic variables among respondents with and without a history of child abuse. Respondents aged 35 to 64 years were more likely than those aged 18 to 34 years to report having been abused as a child. Child abuse was less likely among respondents who were never married than among respondents who were married or in a common-law relationship. The provinces with the highest prevalence of child abuse were Manitoba (40.0\%), British Columbia $(35.8 \%)$ and Alberta $(36.1 \%)$. The province with the lowest prevalence of child abuse was Newfoundland and Labrador (20.5\%).

In the models adjusting for sociodemographic covariates, all child abuse types were associated with increased odds of all mental conditions (Tables 3 and 4). After further adjustment for other child abuse types and mental disorders, most interview-diagnosed mental disorders and several self-reported mental conditions 
remained associated with physical and sexual abuse. However, obsessive-compulsive disorder remained associated only with sexual abuse.
Eating disorders remained associated with only physical abuse. Posttraumatic stress disorder and learning disability remained associated with

Table 3: Odds of interview-diagnosed mental condition from the Composite International Diagnostic Interview, by type of child abuse

Physical abuse

\begin{tabular}{|c|c|c|c|c|c|c|c|}
\hline \multirow[b]{2}{*}{ Mental condition } & \multicolumn{4}{|c|}{ Physical abuse } & \multirow[b]{2}{*}{$\begin{array}{l}\text { Sexual } \\
\text { abuse }\end{array}$} & \multirow[b]{2}{*}{$\begin{array}{l}\text { Exposure to } \\
\text { intimate } \\
\text { partner } \\
\text { violence }\end{array}$} & \multirow[b]{2}{*}{$\begin{array}{l}\text { Any child } \\
\text { abuse }\end{array}$} \\
\hline & $\begin{array}{l}\text { Slapped on } \\
\text { face, head or } \\
\text { ears, hit or } \\
\text { spanked }\end{array}$ & $\begin{array}{l}\text { Pushed, grabbed, } \\
\text { shoved or } \\
\text { something } \\
\text { thrown at }\end{array}$ & $\begin{array}{l}\text { Kicked, bit, } \\
\text { punched, } \\
\text { choked, burned } \\
\text { or attacked }\end{array}$ & $\begin{array}{l}\text { Any physical } \\
\text { abuse }\end{array}$ & & & \\
\hline \multicolumn{8}{|l|}{ Depression } \\
\hline $\begin{array}{l}\text { Adjusted OR1 } \\
(95 \% \mathrm{Cl})\end{array}$ & $2.3(2.0-2.7)$ & $2.8(2.3-3.3)$ & $2.8(2.4-3.3)$ & $2.5(2.2-2.9)$ & $3.2(2.7-3.7)$ & $2.2(1.8-2.8)$ & $2.9(2.5-3.3)$ \\
\hline $\begin{array}{l}\text { Adjusted OR2 } \\
(95 \% \mathrm{Cl})\end{array}$ & $1.3(1.1-1.7)$ & $1.2(0.9-1.6)$ & $1.2(1.0-1.6)$ & $1.6(1.4-2.0)$ & $1.9(1.5-2.4)$ & $0.9(0.6-1.2)$ & $2.2(1.9-2.5)$ \\
\hline \multicolumn{8}{|l|}{ Bipolar disorder } \\
\hline $\begin{array}{l}\text { Adjusted OR1 } \\
(95 \% \mathrm{Cl})\end{array}$ & $2.8(2.1-3.7)$ & $3.7(2.8-5.0)$ & $4.4(3.3-5.9)$ & $3.2(2.5-4.2)$ & $3.6(2.6-4.8)$ & $4.5(3.2-6.3)$ & $3.6(2.7-4.9)$ \\
\hline $\begin{array}{l}\text { Adjusted OR2 } \\
(95 \% \mathrm{Cl})\end{array}$ & $0.9(0.5-1.4)$ & $1.0(0.6-1.8)$ & $1.6(1.0-2.4)$ & $1.2(0.9-1.7)$ & $1.2(0.8-1.8)$ & $2.3(1.3-4.3)$ & $1.9(1.3-2.7)$ \\
\hline \multicolumn{8}{|c|}{ Generalized anxiety disorder } \\
\hline $\begin{array}{l}\text { Adjusted OR1 } \\
(95 \% \mathrm{Cl})\end{array}$ & $2.5(2.1-2.9)$ & $2.9(2.4-3.4)$ & $3.1(2.6-3.7)$ & $2.5(2.2-2.9)$ & $2.8(2.4-3.4)$ & $2.4(2.0-2.9)$ & $2.7(2.3-3.1)$ \\
\hline $\begin{array}{l}\text { Adjusted OR2 } \\
(95 \% \mathrm{Cl})\end{array}$ & $1.4(1.1-1.7)$ & $1.1(0.9-1.5)$ & $1.4(1.1-1.8)$ & $1.5(1.3-1.8)$ & $1.5(1.2-1.9)$ & $1.1(0.8-1.4)$ & $1.8(1.5-2.1)$ \\
\hline \multicolumn{8}{|c|}{ Alcohol abuse/dependence } \\
\hline $\begin{array}{l}\text { Adjusted OR1 } \\
(95 \% \mathrm{Cl})\end{array}$ & $2.4(2.1-2.7)$ & $2.3(2.0-2.7)$ & $2.2(1.9-2.5)$ & $2.5(2.2-2.8)$ & $2.5(2.1-2.9)$ & $2.0(1.7-2.5)$ & $2.5(2.2-2.8)$ \\
\hline $\begin{array}{l}\text { Adjusted OR2 } \\
(95 \% \mathrm{Cl})\end{array}$ & $1.8(1.5-2.1)$ & $1.0(0.8-1.3)$ & $1.1(0.9-1.4)$ & $1.9(1.6-2.2)$ & $1.5(1.2-1.7)$ & $0.9(0.7-1.2)$ & $2.0(1.8-2.2)$ \\
\hline \multicolumn{8}{|c|}{ Drug abuse/dependence } \\
\hline $\begin{array}{l}\text { Adjusted OR1 } \\
(95 \% \mathrm{Cl})\end{array}$ & $3.3(2.8-4.0)$ & $3.4(2.8-4.2)$ & $3.2(2.7-3.9)$ & $3.3(2.8-3.9)$ & $3.8(3.1-4.6)$ & $3.3(2.6-4.1)$ & $3.4(2.9-4.0)$ \\
\hline $\begin{array}{l}\text { Adjusted OR2 } \\
(95 \% \mathrm{Cl})\end{array}$ & $1.7(1.4-2.2)$ & $1.1(0.9-1.5)$ & $1.3(1.0-1.6)$ & $1.8(1.5-2.2)$ & $1.9(1.5-2.4)$ & $1.4(1.1-1.8)$ & $2.3(1.9-2.8)$ \\
\hline \multicolumn{8}{|l|}{ Suicidal ideation } \\
\hline $\begin{array}{l}\text { Adjusted OR1 } \\
(95 \% \mathrm{Cl})\end{array}$ & $3.3(2.9-3.7)$ & $3.7(3.2-4.4)$ & $4.3(3.7-5.0)$ & $3.5(3.1-4.0)$ & $4.6(4.0-5.4)$ & $3.8(3.2-4.5)$ & $4.1(3.7-4.7)$ \\
\hline $\begin{array}{l}\text { Adjusted OR2 } \\
(95 \% \mathrm{Cl})\end{array}$ & $1.6(1.3-1.9)$ & $1.0(0.8-1.2)$ & $1.6(1.3-2.0)$ & $1.8(1.5-2.2)$ & $2.3(1.9-2.8)$ & $1.5(1.2-2.1)$ & $2.9(2.5-3.3)$ \\
\hline \multicolumn{8}{|l|}{ Suicide attempts } \\
\hline $\begin{array}{l}\text { Adjusted OR1 } \\
(95 \% \mathrm{Cl})\end{array}$ & $4.6(3.5-6.0)$ & $5.8(4.5-7.5)$ & $6.6(5.2-8.5)$ & $4.7(3.6-6.2)$ & $7.9(6.1-10.1)$ & $6.3(4.8-8.2)$ & $6.1(4.6-8.0)$ \\
\hline $\begin{array}{l}\text { Adjusted OR2 } \\
(95 \% \mathrm{Cl})\end{array}$ & $1.4(1.0-2.0)$ & $1.0(0.7-1.4)$ & $1.8(1.3-2.6)$ & $1.6(1.1-2.3)$ & $3.0(2.1-4.2)$ & $2.1(1.4-3.2)$ & $3.7(2.7-5.1)$ \\
\hline \multicolumn{8}{|c|}{ Any diagnosis from the Composite International Diagnostic Interview } \\
\hline $\begin{array}{l}\text { Adjusted OR1 } \\
(95 \% \mathrm{Cl})\end{array}$ & $2.8(2.5-3.1)$ & $3.2(2.8-3.8)$ & $3.1(2.7-3.6)$ & $2.9(2.6-3.2)$ & $3.5(3.1-4.1)$ & $2.9(2.4-3.4)$ & $3.1(2.8-3.4)$ \\
\hline $\begin{array}{l}\text { Adjusted OR2 } \\
(95 \% \mathrm{Cl})\end{array}$ & $1.9(1.7-2.2)$ & $1.3(1.0-1.6)$ & $1.5(1.2-1.7)$ & $2.3(2.1-2.6)$ & $2.5(2.1-3.0)$ & $1.3(1.0-1.6)$ & NA \\
\hline \multicolumn{8}{|c|}{$\begin{array}{l}\text { Note: adjusted OR1 = odds ratios adjusted for age, sex, visible minority status, Canadian born status, education, income, marital status and province; adjusted OR2 } \\
\text { (all models except for any physical abuse and child abuse) = odds ratios adjusted for sociodemographic variables listed above, other types of child maltreatment, } \\
\text { and other diagnosed mental disorder from the Composite International Diagnostic Interview; adjusted OR2 (for any physical abuse models) = odds ratios adjusted } \\
\text { for sociodemographic variables listed above, sexual abuse and exposure intimate partner violence, and other diagnosed mental disorder from the Composite } \\
\text { International Diagnostic Interview; adjusted OR2 (for any child abuse models) = odds ratios adjusted for sociodemographic variables listed above and other } \\
\text { diagnosed mental disorder from the Composite International Diagnostic Interview; Cl = confidence interval; NA = not applicable. }\end{array}$} \\
\hline
\end{tabular}


sexual abuse and certain individual types of physical abuse. Bipolar disorder remained associated with exposure to intimate partner vio- lence and one individual type of physical abuse.

All 3 types of abuse remained associated with

drug abuse/dependence, suicidal ideation and

Table 4: Odds of self-reported mental conditions, by type of child abuse

\begin{tabular}{|c|c|c|c|c|c|c|c|}
\hline \multirow[b]{2}{*}{ Mental condition } & \multicolumn{3}{|c|}{ Physical abuse } & \multirow[b]{2}{*}{$\begin{array}{l}\text { Any physical } \\
\text { abuse }\end{array}$} & \multirow[b]{2}{*}{$\begin{array}{l}\text { Sexual } \\
\text { abuse }\end{array}$} & \multirow[b]{2}{*}{$\begin{array}{l}\text { Exposure to } \\
\text { intimate } \\
\text { partner } \\
\text { violence }\end{array}$} & \multirow[b]{2}{*}{$\begin{array}{l}\text { Any child } \\
\text { abuse }\end{array}$} \\
\hline & $\begin{array}{l}\text { Slapped on } \\
\text { face, head } \\
\text { or ears, hit } \\
\text { or spanked }\end{array}$ & $\begin{array}{l}\text { Pushed, grabbed, } \\
\text { shoved or } \\
\text { something } \\
\text { thrown at }\end{array}$ & $\begin{array}{l}\text { Kicked, bit, } \\
\text { punched, } \\
\text { choked, burned } \\
\text { or attacked }\end{array}$ & & & & \\
\hline \multicolumn{8}{|c|}{ Obsessive-compulsive disorder } \\
\hline $\begin{array}{l}\text { Adjusted OR1 } \\
(95 \% \mathrm{Cl})\end{array}$ & $2.6(1.6-4.0)$ & $3.5(2.2-5.4)$ & $3.8(2.4-5.9)$ & $3.1(2.0-4.9)$ & $4.1(2.5-6.6)$ & $3.7(2.3-6.0)$ & $3.0(1.8-4.8)$ \\
\hline $\begin{array}{l}\text { Adjusted OR2 } \\
(95 \% \mathrm{Cl})\end{array}$ & $1.0(0.6-1.6)$ & $1.4(0.8-2.5)$ & $1.3(0.7-2.6)$ & $1.4(0.9-2.2)$ & $1.9(1.2-3.0)$ & $1.4(0.8-2.4)$ & $1.7(1.0-2.9)$ \\
\hline \multicolumn{8}{|l|}{ Panic disorder } \\
\hline $\begin{array}{l}\text { Adjusted OR1 } \\
(95 \% \mathrm{Cl})\end{array}$ & $1.9(1.5-2.4)$ & $2.3(1.8-3.0)$ & $2.8(2.2-3.6)$ & $2.2(1.7-2.7)$ & $2.9(2.3-3.7)$ & $1.8(1.3-2.3)$ & $2.2(1.7-2.8)$ \\
\hline $\begin{array}{l}\text { Adjusted OR2 } \\
(95 \% \mathrm{Cl})\end{array}$ & $1.0(0.7-1.4)$ & $1.1(0.7-1.6)$ & $1.8(1.2-2.5)$ & $1.4(1.1-1.8)$ & $1.7(1.2-2.2)$ & $0.8(0.5-1.1)$ & $1.5(1.2-1.9)$ \\
\hline \multicolumn{8}{|c|}{ Posttraumatic stress disorder } \\
\hline $\begin{array}{l}\text { Adjusted OR1 } \\
(95 \% \mathrm{CI})\end{array}$ & $3.1(2.1-4.5)$ & $5.2(3.6-7.6)$ & $4.9(3.3-7.1)$ & $3.3(2.2-4.8)$ & $7.0(5.1-9.7)$ & $4.5(3.1-6.7)$ & $4.4(3.0-6.3)$ \\
\hline $\begin{array}{l}\text { Adjusted OR2 } \\
(95 \% \mathrm{Cl})\end{array}$ & $0.8(0.5-1.2)$ & $2.2(1.4-3.3)$ & $1.2(0.8-1.9)$ & $1.2(0.8-1.8)$ & $2.9(2.0-4.3)$ & $1.4(0.9-2.2)$ & $2.5(1.7-3.7)$ \\
\hline \multicolumn{8}{|l|}{ Phobias } \\
\hline $\begin{array}{l}\text { Adjusted OR1 } \\
(95 \% \mathrm{Cl})\end{array}$ & $3.2(2.0-5.3)$ & $3.1(1.7-5.8)$ & $3.4(1.9-6.0)$ & $2.9(1.8-4.6)$ & $3.9(2.2-6.9)$ & $3.7(1.9-7.2)$ & $3.2(2.1-5.1)$ \\
\hline $\begin{array}{l}\text { Adjusted OR2 } \\
(95 \% \mathrm{Cl})\end{array}$ & $1.5(1.0-2.4)$ & $1.0(0.6-1.9)$ & $1.0(0.6-1.7)$ & $1.3(0.8-2.0)$ & $2.0(1.1-3.5)$ & $1.8(1.1-3.2)$ & $2.3(1.5-3.6)$ \\
\hline \multicolumn{8}{|c|}{ Attention deficit disorder } \\
\hline $\begin{array}{l}\text { Adjusted OR1 } \\
(95 \% \mathrm{CI})\end{array}$ & $1.7(1.3-2.3)$ & $2.6(1.9-3.6)$ & $2.7(1.9-3.7)$ & $2.1(1.6-2.7)$ & $2.9(2.1-4.0)$ & $1.8(1.2-2.5)$ & $2.2(1.7-2.8)$ \\
\hline $\begin{array}{l}\text { Adjusted OR2 } \\
(95 \% \mathrm{Cl})\end{array}$ & $0.9(0.7-1.2)$ & $1.7(1.1-2.5)$ & $1.5(1.0-2.4)$ & $1.5(1.1-2.0)$ & $1.7(1.2-2.4)$ & $0.8(0.5-1.2)$ & $1.7(1.3-2.2)$ \\
\hline \multicolumn{8}{|l|}{ Eating disorders } \\
\hline $\begin{array}{l}\text { Adjusted OR1 } \\
(95 \% \mathrm{Cl})\end{array}$ & $3.7(2.1-6.7)$ & $3.0(1.5-6.0)$ & $5.2(2.7-10.0)$ & $4.3(2.5-7.6)$ & $4.1(2.2-7.6)$ & $3.4(1.7-6.7)$ & $4.7(2.6-8.5)$ \\
\hline $\begin{array}{l}\text { Adjusted OR2 } \\
(95 \% \mathrm{Cl})\end{array}$ & $1.9(1.0-3.6)$ & $0.5(0.2-1.3)$ & $2.8(1.0-8.0)$ & $2.3(1.2-4.5)$ & $1.6(0.8-3.2)$ & $1.1(0.5-2.5)$ & $3.0(1.7-5.2)$ \\
\hline \multicolumn{8}{|c|}{ Learning disability } \\
\hline $\begin{array}{l}\text { Adjusted OR1 } \\
(95 \% \mathrm{Cl})\end{array}$ & $1.4(1.1-1.8)$ & $2.1(1.6-2.8)$ & $2.6(2.0-3.4)$ & $1.6(1.3-2.0)$ & $2.3(1.7-3.1)$ & $2.3(1.6-3.3)$ & $1.7(1.3-2.1)$ \\
\hline $\begin{array}{l}\text { Adjusted OR2 } \\
(95 \% \mathrm{Cl})\end{array}$ & $0.7(0.5-0.9)$ & $1.3(0.9-1.9)$ & $1.8(1.2-2.7)$ & $1.1(0.8-1.4)$ & $1.5(1.1-2.1)$ & $1.3(0.9-2.1)$ & $1.4(1.1-1.7)$ \\
\hline \multicolumn{8}{|c|}{ Any self-reported mental condition } \\
\hline $\begin{array}{l}\text { Adjusted OR1 } \\
(95 \% \mathrm{Cl})\end{array}$ & $2.1(1.7-2.4)$ & $2.9(2.4-3.5)$ & $3.2(2.6-3.9)$ & $2.2(1.9-2.6)$ & $3.4(2.8-4.1)$ & $2.5(2.0-3.1)$ & $2.3(2.0-2.7)$ \\
\hline $\begin{array}{l}\text { Adjusted OR2 } \\
(95 \% \mathrm{Cl})\end{array}$ & $1.0(0.8-1.1)$ & $1.4(1.1-1.8)$ & $1.6(1.2-2.0)$ & $1.3(1.1-1.5)$ & $1.9(1.6-2.4)$ & $1.1(0.9-1.4)$ & $1.7(1.5-2.0)$ \\
\hline \multicolumn{8}{|c|}{$\begin{array}{l}\text { Note: adjusted OR1 = odds ratios adjusted for age, sex, visible minority status, Canadian born status, education, income, marital status and province; adjusted OR2 } \\
\text { (all models except for any physical abuse and child abuse) = odds ratios adjusted for sociodemographic variables listed above, other types of child abuse and any } \\
\text { diagnosed mental disorders from the Composite International Diagnostic Interview; adjusted OR2 (for any physical abuse models) = odds ratios adjusted for } \\
\text { sociodemographic variables listed above, sexual abuse and exposure intimate partner violence, and any diagnosed mental disorders from the Composite } \\
\text { International Diagnostic Interview; adjusted OR2 (for any child abuse models) = odds ratios adjusted for sociodemographic variables listed above and any } \\
\text { diagnosed mental disorders from the Composite International Diagnostic Interview; } \mathrm{Cl}=\text { confidence interval. }\end{array}$} \\
\hline
\end{tabular}


suicide attempts. There was a general trend of increasing number of child abuse types experienced corresponding with greater odds of mental conditions, indicating a dose-response relation (Table 5).

Significant sex-related effects were only noted between attention deficit disorder and exposure to intimate partner violence (adjusted
OR for the interaction term: $2.0,95 \%$ CI 1.0 4.1) and any child abuse (adjusted OR for the interaction term: $1.8,95 \%$ CI 1.0-3.1), with effects being greater for women. Sex-related effects were found for several child abuse types and suicidal ideation and suicide attempts, with higher prevalence noted among women in all cases (Table 6).

Table 5: Odds of mental conditions, by number of child abuse types

\begin{tabular}{|c|c|c|c|c|}
\hline \multirow[b]{2}{*}{ Mental condition } & \multicolumn{4}{|c|}{ Adjusted OR $(95 \% \mathrm{Cl}) ;{ }^{*}$ number of child abuse types } \\
\hline & No abuse & 1 type & 2 types & 3 types \\
\hline \multicolumn{5}{|c|}{ Interview-diagnosed mental disorders } \\
\hline Depression & 1.0 & $2.6^{\mathrm{a}}(2.2-3.0)$ & $3.2^{\mathrm{a}}(2.6-4.0)$ & $5.3^{b}(3.9-7.0)$ \\
\hline Bipolar & 1.0 & $2.3^{\mathrm{a}}(1.6-3.2)$ & $6.2^{\mathrm{b}}(4.1-9.3)$ & $8.0^{\mathrm{b}}(5.1-12.6)$ \\
\hline Generalized anxiety disorder & 1.0 & $2.2^{\mathrm{a}}(1.8-2.6)$ & $3.0^{b}(2.5-3.7)$ & $5.9^{c}(4.4-7.9)$ \\
\hline Alcohol abuse/dependence & 1.0 & $2.2^{\mathrm{a}}(1.9-2.5)$ & $3.1^{\mathrm{b}}(2.6-3.7)$ & $4.5^{c}(3.3-6.2)$ \\
\hline Drug abuse/dependence & 1.0 & $2.6^{\mathrm{a}}(2.2-3.1)$ & $4.7^{b}(3.6-6.0)$ & $10.8^{c}(7.8-15.0)$ \\
\hline Any mental disorder & 1.0 & $2.6^{\mathrm{a}}(2.3-2.9)$ & $4.0^{b}(3.3-4.7)$ & $8.3^{c}(6.2-11.0)$ \\
\hline \multicolumn{5}{|l|}{ Suicide } \\
\hline Suicidal ideation & 1.0 & $3.2^{\mathrm{a}}(2.7-3.7)$ & $5.0^{b}(4.1-6.0)$ & $13.8^{c}(10.4-18.2)$ \\
\hline Suicide attempts & 1.0 & $3.4^{\mathrm{a}}(2.4-4.8)$ & $7.5^{b}(5.5-10.3)$ & $27.2^{c}(18.2-40.6)$ \\
\hline \multicolumn{5}{|l|}{ Self-reported mental conditions } \\
\hline Obsessive-compulsive disorder & 1.0 & $1.6^{\mathrm{a}}(0.9-2.8)$ & $4.5^{\mathrm{b}} \quad(2.5-8.2)$ & $8.9^{b}(4.3-18.2)$ \\
\hline Panic disorder & 1.0 & $1.8^{\mathrm{a}}(1.3-2.3)$ & $2.5^{\mathrm{b}} \quad(1.8-3.4)$ & $4.4^{c}(3.1-6.3)$ \\
\hline Posttraumatic stress disorder & 1.0 & $2.5^{\mathrm{a}}(1.5-4.1)$ & $5.2^{\mathrm{b}} \quad(3.3-8.1)$ & $16.6^{c}(9.8-28.2)$ \\
\hline Phobias & 1.0 & $2.2^{\mathrm{a}}(1.3-3.6)$ & $3.0^{\mathrm{a}} \quad(1.7-5.2)$ & $9.9^{b}(4.0-24.6)$ \\
\hline Attention-deficit disorder & 1.0 & $1.9^{\mathrm{a}}(1.4-2.5)$ & $2.5^{\mathrm{a}, \mathrm{b}}(1.8-3.5)$ & $4.0^{\mathrm{b}}(2.3-6.9)$ \\
\hline Eating disorders & 1.0 & $3.6^{\mathrm{a}}(1.7-7.5)$ & $4.7^{\mathrm{a}, \mathrm{b}}(2.1-10.6)$ & $10.8^{b}(4.3-27.1)$ \\
\hline Learning disability & 1.0 & $1.2^{\mathrm{a}}(0.9-1.5)$ & $2.5^{\mathrm{b}} \quad(1.7-3.6)$ & $3.2^{\mathrm{b}}(2.0-5.1)$ \\
\hline Any self-reported mental disorder & 1.0 & $1.7^{\mathrm{a}}(1.4-2.0)$ & $2.8^{\mathrm{b}} \quad(2.3-3.5)$ & $6.8^{\mathrm{c}}(4.8-9.7)$ \\
\hline
\end{tabular}

Table 6: Interaction terms between sex and type of abuse for associations with suicidal ideation and suicide attempts

\begin{tabular}{|c|c|c|}
\hline \multirow[b]{2}{*}{ Type of abuse } & \multicolumn{2}{|c|}{ Adjusted OR $(95 \% \mathrm{Cl})$, interaction terms* } \\
\hline & Suicidal ideation & Suicide attempts \\
\hline Slapped on face, head or ears, hit or spanked & $1.5(1.1-1.9)$ & $1.7(1.0-2.7)$ \\
\hline Pushed, grabbed, shoved or something thrown at & $1.3(0.98-1.8)$ & $2.0(1.3-4.0)$ \\
\hline Sexual abuse & $1.3(0.9-1.8)$ & $1.5(0.9-2.6)$ \\
\hline Exposure to intimate partner violence & $1.0(0.7-1.5)$ & $1.2(0.6-2.1)$ \\
\hline Any child abuse & $1.6(1.2-2.0)$ & $1.8(1.0-3.1)$ \\
\hline
\end{tabular}




\section{Interpretation}

We found strong associations between child abuse and mental conditions. In Canada, 32\% of the adult population has experienced physical abuse, sexual abuse and/or exposure to intimate partner violence in childhood. All 3 types of child abuse were associated with all types of interview-diagnosed mental disorders, selfreported mental conditions, suicidal ideation and suicide attempts in models adjusting for sociodemographic variables. These models indicate that the relation between all 3 types of child abuse and mental conditions are robust. The strongest findings were for drug abuse/ dependence, suicidal ideation and suicide attempts, with all 3 types of child abuse remaining significantly associated with these outcomes in the most adjusted models. For other mental conditions in the most adjusted models adjusting for co-occurring types of child abuse and comorbid mental disorders, specific associations were noted between certain types of child abuse and certain mental health disorders. We found a dose-response relation, with a trend of more types of child abuse corresponding with greater odds of mental conditions. Associations of some child abuse types with suicidal ideation and suicide attempts were stronger among women than men.

Although measurement and assessment of child abuse across studies are not directly comparable, the prevalence of child abuse in Canada reported in this study is similar to findings from national surveys from the United States and provincial findings from Ontario and Quebec. ${ }^{2-5,7,11}$ The current findings are also consistent with previous studies that found robust relations with specific types of child abuse and mental conditions, including suicidal ideation and suicide attempts. ${ }^{5-7,10,13,16,18,19}$ What the current study adds is the assessment of a greater number of child abuse types along with more mental conditions in one study. Importantly, the least severe type of physical abuse (being slapped on the face, head or ears, or hit or spanked with something hard) showed a strong association with all mental conditions in models adjusting for sociodemographic covariates.

\section{Strengths and limitations}

Strengths of the study include: the data are current and nationally representative at the provincial level in Canada, the large sample allowed for several sophisticated and high-powered models to be conducted, and several types of child abuse and mental conditions were assessed.

However, the data from this study are crosssectional and retrospective in nature. Inferences regarding causation cannot be made, and underlying mechanisms in the relation are not clear from these data. It may be that for some people a direct relation does exist between child abuse and mental disorders. It may also be the case that behavioural issues for some children could be associated with mental disorders and that behavioural problems could increase the likelihood of child abuse. Longitudinal data are necessary to clarify the possible underlying mechanisms in the association between child abuse and mental disorders. With regard to the retrospective nature of the data, there is debate about accurate recall of traumatic memories. Recall of sexual abuse in particular is controversial. However, evidence suggests that retrospective recall of adverse childhood events provides valid and reliable survey data. ${ }^{25-27}$

Three of the major types of child maltreatment were assessed, but measures of neglect and emotional abuse were not included in the survey. Canadian data on these additional types of child maltreatment are needed.

Although several mental conditions were assessed in the survey, many important disorders (e.g., personality disorders) were not included, and several disorders were assessed using selfreported diagnoses. Self-reported data to determine prevalence of mental conditions has been used in other studies $;{ }^{28}$ it is considered a reliable approach for assessment in community samples ${ }^{29}$ albeit with limitations. The self-reported mental conditions included here are considered to be less biased because they were based on self-reported diagnosis by a health professional.

\section{Conclusion}

Our findings indicate that $32 \%$ of the adult population in Canada has experienced child abuse (i.e., physical abuse, sexual abuse and/or exposure to intimate partner violence) and that child abuse has robust associations with mental conditions. Child abuse is an important public health problem globally, including in Canada. All health care providers should be aware of the relation between specific types of child abuse and certain mental conditions. Clinicians working in the mental health field should be skilled in assessing patients for exposure to abuse and should understand the implications for treatment. In addition, all clinicians need to be familiar with the mandatory reporting requirements for their province or territory. From a public health standpoint, these findings highlight the urgent need to make prevention of child abuse a priority in Canada. Success in preventing child abuse could lead to reductions in the prevalence of mental disorders. 


\section{References}

1. MacMillan HL, Fleming JE, Trocmé N, et al. Prevalence of child physical and sexual abuse in the community. Results from the Ontario Health Supplement. JAMA 1997;278:131-5.

2. MacMillan HL, Tanaka M, Duku E, et al. Child physical and sexual abuse in a community sample of young adults. Results from the Ontario Child Health Study. Child Abuse Negl 2013;37: $14-21$.

3. Tourigny M, Hebert M, Joly J, et al. Prevalence and cooccurence of violence against children in the Quebec population. Aust N Z J Public Health 2008;32:331-5.

4. Hébert M, Tourigny M, Cyr M, et al. Prevalence of childhood sexual abuse and timing of disclosure in a representative sample of adults from Quebec. Can J Psychiatry 2009;54:631-6.

5. Afifi TO, Enns MW, Cox BJ, et al. Population attributable fractions of psychiatric disorders and suicidal ideation and attempts associated with adverse childhood events in the general population. Am J Public Health 2008;98:946-52.

6. Afifi TO, Brownridge DA, Cox BJ, et al. Physical punishment, childhood abuse, and psychiatric disorders. Child Abuse Negl 2006;30:1093-103.

7. MacMillan HL, Fleming JE, Streiner DL, et al. Childhood abuse and lifetime psychopathology in a community sample. Am J Psychiatry 2001;158:1878-83.

8. Scott KM, Smith DR, Ellis PM. Prospectively ascertained child maltreatment and its association with DSM-IV mental disorders in young adults. Arch Gen Psychiatry 2010;67:712-9.

9. Kessler RC, McLaughlin KA, Green JG, et al. Childhood adversities and adult psychopathology in the WHO World Mental Health Surveys. Br J Psychiatry 2010;197:378-85.

10. Afifi TO, Mota NP, Dasiewicz P, et al. Physical punishment and mental disorders: results from a nationally representative US sample. Pediatrics 2012;130:184-92.

11. Afifi TO, Mather A, Boman J, et al. Childhood adversity and personality disorders: results from a nationally representative population-based study. J Psychiatr Res 2011;45:814-22.

12. Afifi TO, Cox BJ, Martens PJ, et al. The relationship between problem gambling and mental and physical health correlates among a nationally representative sample of Canadian women. Can J Public Health 2010;101:171-5.

13. Afifi TO, Boman J, Fleisher W, et al. The relationship between child abuse, parental divorce, and lifetime mental disorders and suicidality in a nationally representative adult sample. Child Abuse Negl 2009;33:139-47.

14. Afifi TO, Enns MW, Cox BJ, et al. Child abuse and healthrelated quality of life. J Nerv Ment Dis 2007;195:797-804.

15. Bruffaerts R, Demyttenaere K, Borges G, et al. Childhood adversities as risk factors for onset and persistence of suicida behaviour. Br J Psychiatry 2010;197:20-7.

16. Enns MW, Cox BJ, Afifi TO, et al. Childhood adversities and risk for suicidal ideation and attempts: a longitudinal populationbased study. Psychol Med 2006;36:1769-78.

17. Fergusson DM, McLeod GFH, Horwood LJ. Childhood sexual abuse and adult developmental outcomes: findings from a 30year longitudinal study in New Zealand. Child Abuse Negl 2013; 37:664-74.

18. Kessler RC, Davis CG, Kendler KS. Childhood adversity and adult psychiatric disorder in the US National Comorbidity Sur vey. Psychol Med 1997;27:1101-19.

19. MacMillan HL, Boyle MH, Wong MYY, et al. Slapping and spanking in childhood and its association with lifetime prevalence of psychiatric disorders in a general population sample. CMAJ 1999;161:805-9.

20. Canadian Community Health Survey (CCHS) - Mental Health user guide. Ottawa $(\mathrm{ON})$ : Statistics Canada, 2013.

21. Canadian Community Health Survey (CCHS) - Mental Health Questionnaire - November 30, 2011. Ottawa (ON): Statistics
Canada, 2011. Available: www23.statcan.gc.ca/imdb-bmdi /instrument/5105_Q1_V3-eng.pdf (accessed 2014 Mar. 24).

22. Walsh CA, MacMillan HL, Trocmé N, et al. Measurement of victimization in adolescence: development and validation of the Childhood Experiences of Violence Questionnaire. Child Abuse Negl 2008;32:1037-57.

23. Diagnostic and statistical manual of mental disorders. Fourth edition. Text revision. Washington (DC): American Psychiatric Association; 2000

24. Canadian Community Health Survey: Mental Health and WellBeing Derived Variable (DV) Specifications. Ottawa (ON): Statistics Canada; 2013

25. Hardt J, Vellaisamy P, Schoon I. Sequelae of prospective versus retrospective reports of adverse childhood experiences. Psychol Rep 2010;107:425-40.

26. Hardt J, Rutter M. Validity of adult retrospective reports of adverse childhood experiences: review of the evidence. $J$ Child Psychol Psychiatry 2004;45:260-73.

27. Hardt J, Sidor A, Bracko M, et al. Reliability of retrospective assessments of childhood experiences in Germany. J Nerv Ment Dis 2006;194:676-83.

28. Sareen J, Cox BJ, Stein MB, et al. Physical and mental comorbidity, disability, and suicidal behavior associated with posttraumatic stress disorders in a large community sample. Psychosom Med 2007;69:242-8.

29. Keski-Rahkonen A, Silhvola E, Raevuori A, et al. Reliability of self-reported eating disorders: optimizing population screening. Int J Eat Disord 2006;39:754-62.

Affiliations: Departments of Community Health Sciences (Afifi, Sareen), Psychiatry (Afifi, Sareen), Family Social Sciences (Afifi), Applied Health Sciences (Taillieu), Psychology (Cheung, Sareen). University of Manitoba, Winnipeg, Man.; Department of Psychiatry and Behavioural Neurosciences (MacMillan, Boyle), Department of Pediatrics (MacMillan), McMaster University, Hamilton, Ont.

Contributors: Tracie Afifi developed the research questions, designed and supervised the statistical analysis, interpreted the findings and wrote sections of the manuscript. Harriet MacMillan and Michael Boyle assisted with the development of the research questions, interpreted the findings and wrote sections of the manuscript. Tamara Taillieu and Kristene Cheung developed the research questions, designed and conducted the statistical analysis, and revised the manuscript. Jitender Sareen developed the research questions, designed the statistical analysis, interpreted the findings and revised the manuscript. All of the authors approved the final version submitted for publication.

Funding: Preparation of this article was supported by a Canadian Institutes of Health Research (CIHR) operating grant (Afifi, MacMillan, Boyle and Sareen), a CIHR New Investigator award (Afifi), a Manitoba Health Research Council establishment grant (Afifi) and a Manitoba Health Research Council Chair Award (Sareen). Harriet MacMillan is supported by the Chedoke Health Chair in Child Psychiatry. Michael Boyle is a Canada Research Chair in the Social Determinants of Child Health.

Acknowledgement: Statistics Canada collected and provided the data for academic purposes, but the analyses are the sole responsibility of the authors. The opinions expressed do not represent the views of Statistics Canada. 